\title{
AN APPROXIMATION METHOD FOR WIENER INTEGRALS OF CERTAIN UNBOUNDED FUNCTIONALS
}

\author{
HENRY C. FINLAYSON
}

Introduction. In Cameron's paper [1] there appears a "rectangle formula" (there denoted by Theorem 1) by means of which the Wiener integral of $F[x(\cdot)]$, defined on the space of continuous functions on $[0,1]$ such that $x(0)=0$, can be approximated by means of an $n$-fold Riemann integral provided $F$ is sufficiently smooth and dominated by a suitable integrable functional. The formula employs a particular C.O.N. set of functions $\left\{\alpha_{i}(s)\right\}^{1}$ (the odd harmonic cosine functions.) A generalization of this formula appears in Cameron's unpublished notes and appears with proof in the author's [2], (there denoted by Theorem 2). The generalization allows for the use of an arbitrary C.O.N. set $\left\{\alpha_{i}(s)\right\}$ of B.V. but suffers the defect that it is not known to hold except for bounded functionals. It is the purpose of this paper to show that if the $\left\{\alpha_{i}(s)\right\}$ 's of this generalization are restricted to certain Sturm-Liouville sets of functions (among which sets is included the odd harmonic cosine functions), then the restricted generalization is applicable to suitably dominated (not necessarily bounded) functionals.

Let $w(s)>0$ have continuous fourth derivative on $[0,1]$ and let $\left\{\lambda_{i}\right\}$ and $\left\{\alpha_{i}(s)\right\}$ be the sets of characteristic numbers and normalized functions of the Sturm-Liouville problem: $\left\{[1 / w(s)] \alpha^{\prime}(s)\right\}^{\prime}$ $+\lambda \alpha(s)=0, \alpha^{\prime}(0)=\alpha(1)=0$ on $[0,1]$. Let $C$ be the space of continuous functions $x(s)$ on $[0,1]$ such that $x(0)=0$, let $\beta_{i}(s)=\int_{0}^{s} \alpha_{i}(t) d t$ and for $x(\cdot) \in C$ let $c_{i}=\int_{0}^{1} \alpha_{i}(s) d x(s): i=1,2,3, \cdots \quad\left(c_{i}\right.$ is of course a functional of $x(\cdot))$. The main result of the paper is the following.

THEOREM. Let $F[x(\cdot)]$ be continuous in the Hilbert topology on $C$ and let

$$
|F[x(\cdot)]| \leqq H\left(\int_{0}^{1} w(t) x^{2}(t) d t\right) \text { on } C,
$$

where $H(u)$ is monotonically increasing on $[0,1]$ and

$$
\int_{c} H\left(\int_{0}^{1} w(t) x^{2}(t) d t\right) d x<\infty
$$

Received by the editors August 25, 1966.

1 The $\beta$ 's of Cameron's paper [1] correspond to the $\alpha$ 's of this paper, and the $\alpha$ 's of Cameron's paper are constant multiples of the $\beta$ 's of this paper. 
Then

$$
\lim _{n \rightarrow \infty} \int_{c} F\left[\sum_{i=1}^{n} c_{i} \beta_{i}(\cdot)\right] d x=\int_{c} F[x(\cdot)] d x .
$$

It can easily be verified by letting $w(t) \equiv 1$ that Cameron's original rectangle formula is a special case of the theorem.

In $\S 1$ will be given five lemmas and by means of them a proof of the theorem in $\$ 2$.

\section{Five lemmas.}

LEMMA 1.1. If $\left\{f_{i}(s)\right\}$ is a complete set of functions on $[0,1]$ then so also is $\left\{\int_{0}^{s} f_{i}(t) d t\right\}$.

Proof. If for $g(s) \in L_{2}[0,1]$,

$$
\int_{0}^{1} g(s) \int_{0}^{s} f_{i}(t) d t d s=0, \quad i=1,2,3, \cdots,
$$

then by interchanging the order of integration there follows

$$
\int_{0}^{1} \int_{t}^{1} g(s) d s f_{i}(t) d t=0, \quad i=1,2,3, \cdots,
$$

so that $\int_{t}^{1} g(s) d s$, and hence $g(s)$ itself, equals zero almost everywhere.

LEMMA 1.2. If $\left\{f_{i}(s)\right\}$ is a complete set of functions on $[0,1]$ and if $h(s)$ is continuous and $h(s)>0$ on $[0,1]$ then $\left\{h(s) f_{i}(s)\right\}$ is a complete set of functions on $[0,1]$.

Proof. There will be shown that any $g(s) \in L_{2}[0,1]$ can be approximated arbitrarily closely in the $L_{2}$ sense by a linear combination of the $h(s) f_{i}(s)$ 's. Let $a_{i}, i=1,2,3, \cdots, n$, be unspecified constants.

$$
\begin{aligned}
\int_{0}^{1}\left[g(s)-\sum_{i=1}^{n}\right. & \left.a_{i} h(s) f_{i}(s)\right]^{2} d s \\
& =\int_{0}^{1}[h(s)]^{2}\left[g(s) / h(s)-\sum_{i=1}^{n} a_{i} f_{i}(s)\right]^{2} d s \\
& \leqq\left[\max _{0 \leqq s} h(s)\right]^{2} \int_{0}^{1}\left[g(s) / h(s)-\sum_{i=1}^{n} a_{i} f_{i}(s)\right]^{2} d s
\end{aligned}
$$

Now since $g(s) / h(s) \in L_{2}[0,1]$, suitable $n$ and $a_{i}$ 's can be found to make the integral in the last member of (1.1) arbitrarily small. This completes the proof of the lemma.

LeMmA 1.3. Let $w(s)>0$ be continuous on $[0,1]$. If $\lambda_{i}$ and $\alpha_{i}(s)$ are 
the characteristic numbers and normal functions respectively of the Sturm-Liouville problem: $\left\{[1 / w(s)] \alpha^{\prime}(s)\right\}^{\prime}+\lambda \alpha(s)=0, \alpha^{\prime}(0)=\alpha(1)=0$ on $[0,1]$, then $\left\{\sqrt{ }\left[\lambda_{i} w(s)\right] \beta_{i}(s)\right\}$ is a complete orthonormal set of functions on $[0,1]$.

Proor. From the theory of Sturm-Liouville problems e.g. Ince $[3,241]$ it is known that the characteristic numbers occur with multiplicity one and that to each characteristic number corresponds just one (independent) characteristic function. Also, a result similar to $[3,237-238,273-276]$ is that the normalized characteristic functions constitute a C.O.N. set of functions. That all the characteristic numbers are positive will now be shown. One integration of the Sturm-Liouville equation yields

$$
\beta_{i}(s)=-\alpha_{i}^{\prime}(s) /\left[\lambda_{i} w(s)\right] .
$$

If both sides of (1.2) are multiplied by $\lambda_{i} w(s) \beta_{i}(s)$ and integrated there follows

$$
\begin{aligned}
\lambda_{i} \int_{0}^{1} w(s) \beta_{i}^{2}(s) d s & =-\int_{0}^{1} \beta_{i}(s) \alpha_{i}^{\prime}(s) d s \\
& =-\left.\beta_{i}(s) \alpha_{i}(s)\right|_{0} ^{1}+\int_{0}^{1} \alpha_{i}^{2}(s) d s=1,
\end{aligned}
$$

(because $\left.\beta_{i}(0)=\alpha_{i}(1)=0\right)$ and thus $\lambda_{i}$ is positive.

Since $\left\{\alpha_{i}(s)\right\}$ is C.O.N. there follows from Lemmas 1.1 and 1.2 that $\left\{\sqrt{ }\left[\lambda_{i} w(s)\right] \beta_{i}(s)\right\}$ is a complete set of functions.

There need only be verified that the latter is an orthonormal set. But from (1.2) follows

$$
\begin{aligned}
\int_{0}^{1} & \sqrt{ }\left[\lambda_{i} w(s)\right] \beta_{i}(s) \sqrt{ }\left[\lambda_{j} w(s)\right] \beta_{j}(s) d s \\
& =-\left(\lambda_{i} / \lambda_{j}\right)^{1 / 2} \int_{0}^{1} \beta_{i}(s) \alpha_{j}^{\prime}(s) d s \\
& =-\left(\lambda_{i} / \lambda_{j}\right)^{1 / 2}\left[\left.\beta_{i}(s) \alpha_{j}(s)\right|_{0} ^{1}-\int_{0}^{1} \alpha_{j}(s) \alpha_{i}(s) d s\right] \\
& \left.=\left(\lambda_{i} / \lambda_{j}\right)^{1 / 2} \int_{0}^{1} \alpha_{i}(s) \alpha_{j}(s) d s \quad \quad \text { (because } \beta_{i}(0)=\alpha_{i}(1)=0\right) \\
& =\delta_{i j} \quad
\end{aligned}
$$

which completes the required verification. 
LEMma 1.4.

$$
\int_{0}^{1} w(t)\left[\sum_{i=1}^{n} c_{i} \beta_{i}(t)\right]^{2} d t=\sum_{i=1}^{n} c_{i}^{2} / \lambda_{i} \leqq \int_{0}^{1} w(t) x^{2}(t) d t
$$

(1.4) $\lim _{n \rightarrow \infty} \int_{0}^{1} w(t)\left[\sum_{i=1}^{n} c_{i} \beta_{i}(t)\right]^{2} d t=\sum_{i=1}^{\infty} c_{i}^{2} / \lambda_{i}=\int_{0}^{1} w(t) x^{2}(t) d t$.

Proof. (1.3) and (1.4) are respectively Bessel's inequality and Parseval's equation for the development of $[w(t)]^{1 / 2} x(t)$ with respect to the C.O.N. set $\left\{\left[\lambda_{i} w(t)\right]^{1 / 2} \beta_{i}(t)\right\}$. The verification of this assertion follows.

$$
\begin{aligned}
& \int_{0}^{1}[w(t)]^{1 / 2} x(t)\left[\lambda_{i} w(t)\right]^{1 / 2} \beta_{i}(t) d t \\
& =-\int_{0}^{1}[w(t)]^{1 / 2} x(t)\left[\lambda_{i} w(t)\right]^{1 / 2} \alpha_{i}^{\prime}(t) /\left[\lambda_{i} w(t)\right] d t
\end{aligned}
$$

(because of (1.2))

$$
\begin{aligned}
& =-\int_{0}^{1} x(t) \alpha_{i}^{\prime}(t) /\left(\lambda_{i}\right)^{1 / 2} d t \\
& =-x(t) \alpha_{i}(t) /\left.\left(\lambda_{i}\right)^{1 / 2}\right|_{0} ^{1}+\int_{0}^{1} \alpha_{i}(t) d x(t) /\left(\lambda_{i}\right)^{1 / 2} \\
& \left.=c_{i} /\left(\lambda_{i}\right)^{1 / 2} \quad \text { (because } x(0)=\alpha_{i}(1)=0\right) .
\end{aligned}
$$

Lemma 1.5.

$$
\lim _{n \rightarrow \infty} \int_{0}^{1}\left[x(t)-\sum_{i=1}^{n} c_{i} \beta_{i}(t)\right]^{2} d t=0
$$

Proof.

$$
\begin{array}{r}
\int_{0}^{1}\left[\min _{0 \leqq t \leqq 1} w(t)\right]\left[x(t)-\sum_{i=1}^{n} c_{i} \beta_{i}(t)\right]^{2} d t \\
\leqq \int_{0}^{1} w(t)\left[x(t)-\sum_{i=1}^{n} c_{i} \beta_{i}(t)\right]^{2} d t
\end{array}
$$

and the limit of the right side of the inequality is, by Lemma 1.4, equal to zero. Since $\left[\min _{0 \leq t \leq 1} w(t)\right]$ is a constant greater than zero the proof is complete. 
2. The proof of the theorem. Now will be given the proof of the theorem stated in the introduction. First there is observed that because of Lemma 1.4 and the assumption on $F$

$$
\begin{aligned}
\left|F\left[\sum_{i=1}^{n} c_{i} \beta_{i}(\cdot)\right]\right| & \leqq H\left(\int_{0}^{1} w(t)\left[\sum_{i=1}^{n} c_{i} \beta_{i}(t)\right]^{2} d t\right) \\
& \leqq H\left(\int_{0}^{1} w(t) x^{2}(t) d t\right) .
\end{aligned}
$$

Also, from Lemma 1.5 and the continuity of $F$ in the Hilbert topology

$$
\lim _{n \rightarrow \infty} F\left[\sum_{i=1}^{n} c_{i} \beta_{i}(\cdot)\right]=F[x(\cdot)] .
$$

Lebesgue's dominated convergence theorem completes the proof.

Finally there will be shown that there is a nontrivial $H(u)$ satisf $y^{-}$ ing the hypotheses of the theorem. For any $k<\lambda_{0}$ (again note $\lambda_{0}>0$ so that $k$ can be chosen positive) $\exp (k u)$ will serve as $H(u)$. To prove this there is observed that Wiener's formula for functions of $n$ linear functionals, viz.

$$
\begin{aligned}
\int_{c} f\left(c_{1}, \cdots, c_{n}\right) d x \\
\quad=\int_{-\infty}^{\infty}(n) \int_{-\infty}^{\infty} \pi^{-n / 2} \exp \left(-\xi_{1}^{2} \cdots-\xi_{n}^{2}\right) f\left(\xi_{1}, \cdots, \xi_{n}\right) d \xi_{1} \cdots d \xi_{n}
\end{aligned}
$$

yields that

$$
\begin{aligned}
\int_{c} \exp \left(k \int_{0}^{1} w(t)\left[\sum_{i=1}^{n} c_{i} \beta_{i}(t)\right]^{2} d t\right) d x \\
=\int_{c} \exp \left(k \sum_{i=1}^{n} c_{i}^{2} / \lambda_{i}\right) d x \\
=\prod_{i=1}^{n} \int_{-\infty}^{\infty} \pi^{-1 / 2} \exp \left(-\xi_{i}^{2}+k \xi_{i}^{2} / \lambda_{i}\right) d \xi_{i} \\
=\prod_{i=1}^{n}\left\{1 / \sqrt{ }\left[1-k / \lambda_{i}\right]\right\} .
\end{aligned}
$$

The estimate given for $\lambda_{i}$ in $[3,272]$ ensures that the infinite product converges. An appeal to Lebesgue's monotone convergence theorem completes the establishment of the result. 


\section{REFERENCES}

1. R. H. Cameron, A "Simpson's Rule" for the numerical evaluation of Wiener's integrals in function space, Duke Math. J. 18 (1951), 111-130.

2. Henry C. Finlayson, New approximations for Wiener integrals, with error estimates, Canad. J. Math. 19 (1967), 58-105.

3. E. L. Ince, Ordinary differential equations, Dover, New York, 1956.

UNIVERSITY OF MANITOBA

\section{REMARKS ON SOME CONVERGENCE CONDITIONS FOR CONTINUED FRACTIONS}

DAVID F. DAWSON

In [4] Farinha proved the following theorem concerning the continued fraction

$$
\frac{1}{1}+\frac{a_{1}}{1}+\frac{a_{2}}{1}+\frac{a_{3}}{1}+\cdots .
$$

TheOREm. Suppose the $a_{k}$ are functions of a complex variable defined in a region $D$. If the $a_{k}$ satisfy at each point of $D$ the conditions

(i) no term of $\left\{a_{n}\right\}$ is zero, but $\lim _{n} a_{n}=0$,

(ii) $\left|a_{1}\right| \leqq \alpha$ and $\left|1+a_{1}\right| \geqq\left|a_{1}\right|+\mu$, where $\alpha$ and $\mu$ are positive constants, and

(iii) $\left|1+a_{n}+a_{n+1}\right| \geqq 2\left|a_{i}\right|, i=n, n+1 ; n=1,2,3, \cdots$, then the continued fraction (1) converges uniformly over $D$ and the modulus of its value does not exceed the smaller of the numbers $3 / 2$ and $(\alpha+\mu) / \mu^{2}$.

Convergence of (1) under Farinha's three conditions follows from a theorem of Scott and Wall [6, Theorem 3.4].

In this note we show that (iii) alone is sufficient to give convergence of the continued fraction (Theorem 1), and, using the basic idea involved in the proof, we extend a theorem of the author $[3$, Theorem A].

THEOREM 1. If for each positive integer $n,\left|1+a_{n}+a_{n+1}\right| \geqq 2\left|a_{i}\right|$, $i=n, n+1$, then the continued fraction (1) converges.

Proof. From the hypothesis and the triangle inequality we can

Presented to the Society, April 22, 1966; received by the editors May 14, 1966. 